\title{
A Preliminary Randomized Controlled Trial of Different Treatment Regimens for Melancholic Depression
}

Yun Wang, ' Xiaohua Liu, ${ }^{2,3}$ Daihui Peng, (1D ${ }^{2}$ Yan $\mathrm{Wu}^{2}$ Yun'ai Su, $\mathbb{D}^{4}$ jia $X u,{ }^{5}$ Xiancang $\mathrm{Ma}, \mathbb{D}^{6} \mathrm{Yi} \mathrm{Li}^{7}$ Jianfei Shi, ${ }^{8}$ Xiaojing Cheng, ${ }^{9}$ Han Rong, ${ }^{10}$ Yiru Fang $(\mathbb{D})^{1,3,11}$

'Division of Mood Disorders, Shanghai Mental Health Center, Shanghai Jiao Tong University School of Medicine, Shanghai, People's Republic of China; ${ }^{2}$ Department of Psychiatry, Shanghai Mental Health Center, Shanghai Jiao Tong University School of Medicine, Shanghai, People's Republic of China; ${ }^{3}$ Shanghai Key Laboratory of Psychotic Disorders, Shanghai Mental Health Center, Shanghai Jiao Tong University School of Medicine, Shanghai, People's Republic of China; ${ }^{4}$ Department of Psychiatry, Peking University Sixth Hospital, Peking, People's Republic of China; ${ }^{5}$ Department of Psychiatry, Harbin First Specific Hospital, Harbin, People's Republic of China; ${ }^{6}$ Department of Psychiatry, The First Affiliated Hospital of Xi'an Jiao Tong University, Xi'an, People's Republic of China; ${ }^{7}$ Department of Psychiatry, Wuhan Mental Health Center, Wuhan, People's Republic of China; ${ }^{8}$ Department of Psychiatry, Hangzhou Seventh People's Hospital, Hangzhou, People's Republic of China; ${ }^{9}$ Department of Psychiatry, Shandong Mental Health Center, Shandong, People's Republic of China; ${ }^{10}$ Department of Psychiatry, Shenzhen Kangning Hospital, Shenzhen, People's Republic of China; "CAS Center for Excellence in Brain Science and Intelligence Technology, Shanghai, People's Republic of China

Correspondence: Daihui Peng; Yiru Fang Email pdhsh@I26.com;

yirufang@aliyun.com
Background: Fluoxetine, bupropion, cognitive behavioral therapy (CBT), and physical therapies (modified electroconvulsive treatment or repetitive transcranial magnetic stimulation) can be used to manage melancholic depression.

Objective: To compare the efficacy and safety of various treatments in patients with melancholic depression.

Methods: This was a preliminary multicenter randomized controlled trial that included patients with depression in their first or recurrent acute episode between September 2016 and June 2019, and randomized to fluoxetine, fluoxetine+CBT, fluoxetine+bupropion, and fluoxetine+bupropion+brain stimulation. The primary endpoint was the decrease in the 17item Hamilton Depression Rating Scale (17-HDRS). The secondary endpoint included the scores from the Quick Inventory of Depressive Symptomatology (QIDS-SR), QOL-6, and safety. Adverse events (AEs) were monitored. The follow-ups were performed at the end of the 0th, 2nd, 4th, 6th, 8th, and 12th weeks of treatment.

Results: Finally, 113 patients were included in the analyses: fluoxetine $(n=37)$, fluoxetine + CBT ( $\mathrm{n}=27)$, fluoxetine+bupropion $(\mathrm{n}=34)$, and fluoxetine+bupropion+brain stimulation $(\mathrm{n}=15)$. The 17-HDRS and QIDS-SR scores decreased in all four groups (all $\mathrm{P}<0.05)$. There were no differences in the 17-HDRS scores among the four groups at the end of treatment $(\mathrm{P}=0.779)$, except for fluoxetine alone showing a better response regarding selfconsciousness than fluoxetine+bupropion. The QOL-6 scores increased in all four groups. The occurrence of AEs among the four groups showed no significant difference $(\mathrm{P}=0.053)$. Conclusion: This preliminary trial suggests that all four interventions (fluoxetine, fluoxetine + CBT, fluoxetine+bupropion, and fluoxetine+bupropion+brain stimulation) achieved similar response and remission rates in patients with melancholic depression, but that fluoxetine had a better effect on self-consciousness than fluoxetine+bupropion. The safety profile was manageable.

Keywords: major depressive disorder, melancholic depression, fluoxetine, bupropion, cognitive behavioral therapy, brain stimulation

\section{Introduction}

Major depressive disorder (MDD) is a common and well-known type of depressive disorder. The prevalence of MDD worldwide is approximately $6 \%$ per year, with a lifetime prevalence of $20 \%{ }^{1}$ Some patients may have specific subtypes of depression, which may be clinically useful for predicting outcomes and choosing treatment: melancholic depression (melancholia), depression with atypical features, MDD with psychotic features, MDD with catatonia, and MDD with anxious 
distress. $^{1-4}$ The prognosis for MDD is variable. It is unremitting in about $15 \%$ of patients and recurrent in about $35 \%$ of patients, with the risk of recurrence increases with each additional episode of major depression. ${ }^{1-5}$

Subjects with melancholic depression show biological abnormalities than healthy controls. Melancholic depression might be characterized by specific biological changes and it could be associated to more severe abnormalities with respect to non-melancholic depression. ${ }^{6}$ Melancholic MDD requires the following features as part of the standard diagnosis of MDD: lack of interest or pleasure in most or all activities (anhedonia), or lack of reactivity to pleasurable stimuli, and at least three among: distinct quality of depressed mood (experienced differently from the loss of a loved one), symptoms regularly worse in the morning, early morning awakening (at least 2 hours before the usual time of awakening), significant loss of appetite or weight loss, noticeable psychomotor retardation or agitation, and excessive or inappropriate guilt. ${ }^{1-4}$ About $25-30 \%$ of the patients with MDD have melancholic features. ${ }^{1-4}$ Melancholia is associated with more severe depression, increased risk of suicide, increased likelihood of treatment response to pharmacotherapy or electroconvulsive therapy over psychotherapy, nonresponse to placebo interventions, decreased likelihood of response to psychotherapy, and high recurrence rate. ${ }^{1-4}$ Melancholia is associated with more severe depression, increased risk of suicide, and a high recurrence rate. ${ }^{1-4}$

Selective serotonin reuptake inhibitors (SSRIs), including fluoxetine, are the first-line treatment. ${ }^{1-4}$ Fluoxetine has a better effect in melancholic depression than in the non-melancholic types, and the patients with melancholic depression show an earlier response and a higher remission rate to a lower dose of fluoxetine. ${ }^{5}$ Cognitivebehavioral therapy helps alleviate depressive symptoms by questioning and challenging the patients' irrational thinking and wrong attitude towards themselves, the surrounding environment, and the future. ${ }^{6}$ Cognitive behavioral therapy (CBT) can be applied in combination with drugs for the acute episode. ${ }^{28}$ Bupropion is a norepinephrine-dopamine reuptake inhibitor and has a similar treatment effect to SSRI. ${ }^{1-4}$ Bupropion is better in improving some symptoms such as fatigue and drowsiness than certain SSRIs. ${ }^{7}$

The treatment of MDD mainly relies on drugs and psychological intervention. ${ }^{1-4}$ Nevertheless, about $20 \%$ of the patients show little improvement in long-term follow-up studies. ${ }^{1-4}$ Physical therapies, including modified electroconvulsive treatment (MECT) or repetitive transcranial magnetic stimulation (rTMS), can be used to manage the symptoms of depression. ${ }^{8-11}$ The efficacy and acceptability of different ways of physical therapy for depression are different. ${ }^{29}$ Nevertheless, currently, there is no randomized controlled trial (RCT) research that compares various treatments in patients with melancholic depression.

Therefore, the objective of the present study was to compare the efficacy and safety of various treatments in patients with melancholic depression. The results could provide data for the stratified treatment of MDD.

\section{Methods}

\section{Study Design and Participants}

This was a multicenter RCT (the participating hospitals are listed in Supplementary Table S1) that included patients with depression in their first or recurrent acute episode between September 2016 and June 2019. The study was approved by the ethics committees of all the participating centers, and the written informed consent was obtained from the study subjects. This was a preliminary study registered with ClinicalTrials.gov (NCT03219008).

The inclusion criteria were: 1) 18-55 years old; 2) patients with melancholic depression who met the DSM5 diagnostic criteria of MDD and were in major depression episode (MDE); 3) patients in their first or recurrent acute episode; 4) scored $\geq 17$ on the Hamilton depression scale (HAMD-17); 5) did not receive any anti-depression medication, physical or psychological therapies within the past 6 months before being recruited into the study; and 6) did not receive systematic treatment at the participating centers.

The patients were diagnosed with melancholic depression based on a clinical subtype determination by Inventory for Depressive Symptomatology (IDS) scoring of the melancholic and atypical symptoms and HAMD Anxiety somatization factor scoring of the symptom weights. $^{12}$

The exclusion criteria were: 1) patients with severe somatic diseases (history of brain injury or cerebrovascular accident, narrow-angle glaucoma, epilepsy, myocardial infarction, unstable angina pectoris, congestive heart failure, severe liver cirrhosis, acute and chronic renal failure, severe diabetes, aplastic anemia, moderate to severe malnutrition and other severe somatic diseases including 
neurological, heart, liver, renal, endocrine and blood disorders or diseases that could interfere with the study; the abnormal indicators had to be at least twice the upper limit of normal (ULN));2) patients with HAMD-17 item 3 (suicide) $\geq 3$ or patients who attempted suicide within this episode; 3) women in pregnancy or nursing period, or women planning to be pregnant; 4) patients with comorbid psychiatric disorders or psychiatric symptoms, drug abusers (nicotine excluded), patients with history of mania or mild mania present in this episode, or patients with mental retardation, personality disorder or anorexia nervosa/bulimia; or 5) patients with secondary depressive disorder caused by organic lesion or drugs. 6) patients with any contraindications for brain stimulation.

\section{Grouping and Blinding}

The participants were randomized 1:1:1:1 into four treatment groups using a central system programmed and maintained by an independent third-party biostatistician: 1) fluoxetine; 2) fluoxetine+CBT; 3) fluoxetine+bupropion; and 4) fluoxetine +bupropion+brain stimulation. Only the person who administered the questionnaires was blind to grouping.

The treatment regimens and the dosing of fluoxetine and bupropion were determined by the physicians. The drugs used in the study were fluoxetine (Prozac, Eli Lilly, Indianapolis, IN, USA) and bupropion hydrochloride sustained-release tablets (Funing Pharmaceutical Co. Ltd., Shenyang, China). Brain stimulation included MECT or rTMS. MECT was performed ten times a month as a course of treatment. rTMS was performed ten times every two weeks as a course of treatment. The duration of each treatment varied between 8 and 12 weeks.

\section{Data Collection and Measurement of Indicators}

Age, sex, ethnicity, body mass index (BMI), education years, occupation, marital status, the total course of depression, duration of current episode, and severity of depression were collected.

The scales used in the study include the 17-item Hamilton Depression Rating Scale (17-HDRS), ${ }^{13}$ SelfRating version of Quick Inventory of Depressive Symptomatology (QIDS-SR), ${ }^{14}$ and Quality of Life (QOL-6), which are all validated scales for MDD, including their Chinese versions. ${ }^{15,16}$ Adverse events (AEs) were recorded in a $\log$.
The 17-HDRS includes 17 items: 1) depressed emotions; 2) feelings of guilt; 3) suicidal ideations; 4) difficulty in falling asleep; 5) failure in sleeping deeply; 6) early awakening; 7) loss in work and interest; 8) retardant/ slow; 9) agitation; 10) spiritually anxious; 11) somatically anxious; 12) gastrointestinal symptoms; 13) general symptoms; 14) sexual symptoms; 15) hypochondriasis; 16) weight loss; and 17) self-consciousness. The items for the melancholic subtypes are \#1-2-6-7-8-9-12-16.

\section{Endpoints}

The primary endpoint was the decrease in the 17-HDRS scores in the intention-to-treat set. A decrease in the 17HDRS scores $\geq 50 \%$ was considered to be responsive; 17 HDRS total score $\leq 7$ was considered to remission. The secondary endpoint included the scores from QIDS-SR and QOL-6.

\section{Follow-Up}

The duration of follow-up varied between 8 and 12 weeks. The follow-ups were performed at the end of the 0th, 2nd, 4th, 6th, 8th, and 12th weeks of treatment, including the measurement of the indicators for efficacy and safety, 17HDRS, QIDS-SR, QOL-6, AE record, evaluation of clinical symptomatology, blood biochemistry, neurological imaging, and electrophysiological examinations. The allowed time window for follow-ups was \pm 2 days.

\section{Safety}

The AEs included gastrointestinal reactions (such as gastric discomfort, diarrhea, constipation, nausea, vomiting, dry and bitter mouth, and increased appetite), abnormal liver functions, headache and dizziness (dizziness, vertigo, and syncope), fatigue (drowsiness, fatigue, lethargy, and slow response), allergy, tremor (including tremor, shaking hands or feet, feeling tired, myasthenia, night sweat, dyspnea, nervousness, and anxiety), changes in the heart rate (including bradycardia and tachycardia), suicide and self-harm (aggressiveness), and general AEs (bleeding nose, hair loss, pneumonia, and fever).

\section{Statistical Analysis}

As this was a preliminary study, no power analysis was performed.

All data were processed and analyzed using SPSS 22.0 (IBM, Armonk, NY, USA) and GraphPad Prism 6 (GraphPad Software, San Diego, USA). The continuous variables were tested for normal distribution using the 
Kolmogorov-Smirnov test. The continuous variables that followed the normal distribution were presented as means \pm standard deviation and analyzed using ANOVA and post hoc pairwise comparisons with the Bonferroni correction; otherwise, the continuous variables were presented as medians (interquartile range (IQR)) and analyzed using the Kruskal-Wallis test. Changes in the scores within each group were tested using the Wilcoxon signed-rank test. The categorical variables were presented as number (percentage) and analyzed using the chi-square test or Fisher's exact test. All tests were two-sided (except the chi-square test), and P-values $<0.05$ were considered statistically significant.

\section{Results}

\section{Characteristics of the Participants}

A total of 186 patients were screened for eligibility and 30 were excluded. Then, 156 were randomized to the fluoxetine $(n=38)$, fluoxetine+CBT $(n=37)$, fluoxetine+bupropion $(n=42)$, and fluoxetine+bupropion+brain stimulation $(n=39)$. During treatment, 43 participants dropped out. Finally, 113 patients were included in the analyses: fluoxetine $(n=37)$, fluoxetine+CBT $(n=27)$, fluoxetine+bupropion $(n=34)$, and fluoxetine+bupropion+brain stimulation $(n=15)$. Their characteristics are presented in Table 1. There were no differences among the four groups regarding any of the characteristics of the participants.

\section{Efficacy Evaluation}

The 17-HDRS scores decreased in all four groups (all $\mathrm{P}<0.05)$. There were no differences in the 17-HDRS scores among the four groups at the end of treatment $(\mathrm{P}=0.779)$, nor in the response rates (ie, 17-HDRS decreasing by $\geq 50 \%)(\mathrm{P}=0.927)$ or the remission rates $(\mathrm{P}=0.658)$. There was no difference for the total melancholic subscore $(\mathrm{P}=0.692)$, but a difference was observed regarding item \#17 (self-consciousness) $(\mathrm{P}=0.019)$, especially between the fluoxetine and fluoxetine+bupropion groups $(\mathrm{P}=0.010)$.

The QIDS-SR scores decreased in all four groups during the follow-ups. The QOL-6 scores increased in all four groups. There were no differences in the QIDS-SR and QOL-6 among the four groups at the end of treatment (Table 2 and Figure 1).

\section{Safety}

There were no differences in the occurrence of AEs among the four groups $(\mathrm{P}=0.053)$. A higher rate of headaches could be observed in the fluoxetine+bupropion+brain stimulation group $(\mathrm{P}=0.035)$ (Table 3$)$.

\section{Discussion}

Fluoxetine, bupropion, CBT, and physical therapies can be used to manage melancholic depression. ${ }^{5-11}$ The aim of the present preliminary RCT was to compare the efficacy and safety of various treatments in patients with melancholic depression. The results suggest that fluoxetine, fluoxetine+CBT, fluoxetine+bupropion, and fluoxetine +bupropion+physical therapies achieved similar response and remission rates, and the occurrence of AEs showed no difference among the four groups but compared with fluoxetine+bupropion, fluoxetine alone had a better effect on self-consciousness, a component of 17-HDRS, but not of the melancholic subscore.

Fluoxetine is among the first-line drugs for the treatment of MDD, with proved efficacy and safety. ${ }^{5,17}$ Its efficacy is superior to that of nortriptyline ${ }^{18}$ and similar to that of sertraline and moclobemide. ${ }^{19,20}$ The combination of fluoxetine with other drugs can lead to higher remission rates in patients with MDD. ${ }^{21}$ Fluoxetine can be combined with bupropion, and this combination has been shown to be effective and safe. ${ }^{7,22} \mathrm{CBT}$ incorporates modifying and refocusing dysfunctional beliefs (cognitive restructuring) to impact behavior and functioning. ${ }^{1} \mathrm{CBT}$ alone has some efficacy in MDD, but its efficacy is considered lower in patients with melancholic MDD, ${ }^{3,4}$ and it is therefore often used in combination with drugs. ${ }^{6,23,24}$ MECT and rTMS can be applied in the management of the symptoms of depression. ${ }^{8-11}$

The use of a single drug or treatment modality often results in a suboptimal response, and combinations are often required. ${ }^{25}$ The present study compared fluoxetine vs fluoxetine+CBT vs fluoxetine+bupropion vs fluoxetine+bupropion+physical therapies. All four methods decreased the symptoms of MDD and improved quality of life. Nevertheless, there were no differences among the four groups. CBT did not improve the response when added to fluoxetine, but it is known that patients with melancholic MDD have a lower response to CBT. $^{3,4}$ In addition, melancholic MDD has a high response rate to SSRIs (like fluoxetine) and MECT, ${ }^{3,4}$ which might explain, at least in part, the lack of difference between fluoxetine alone vs fluoxetine+bupropion and fluoxetine+bupropion+physical therapies. Selfconsciousness is a type of hypervigilance state that is associated with obsessive-compulsive disorders, as well 


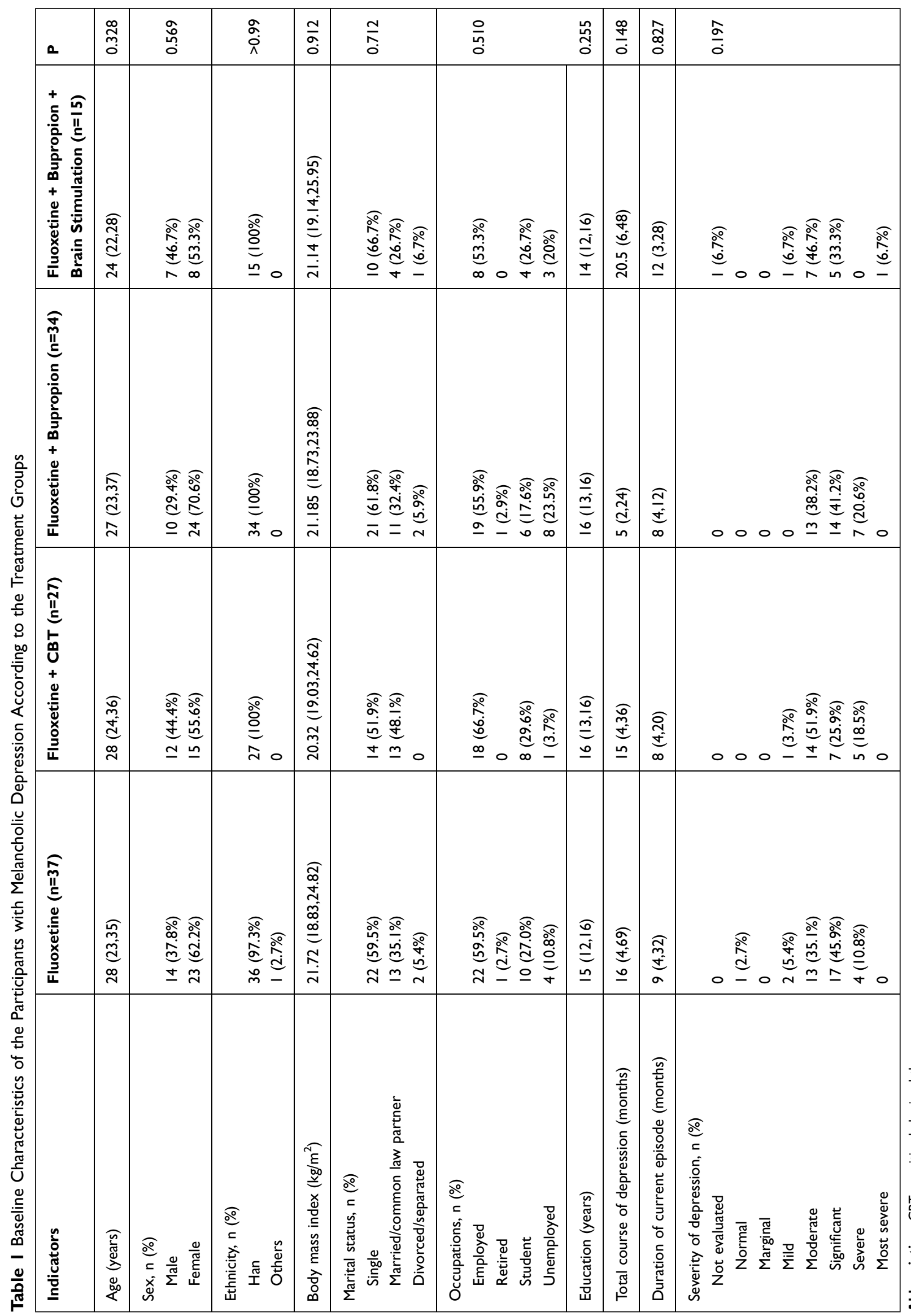




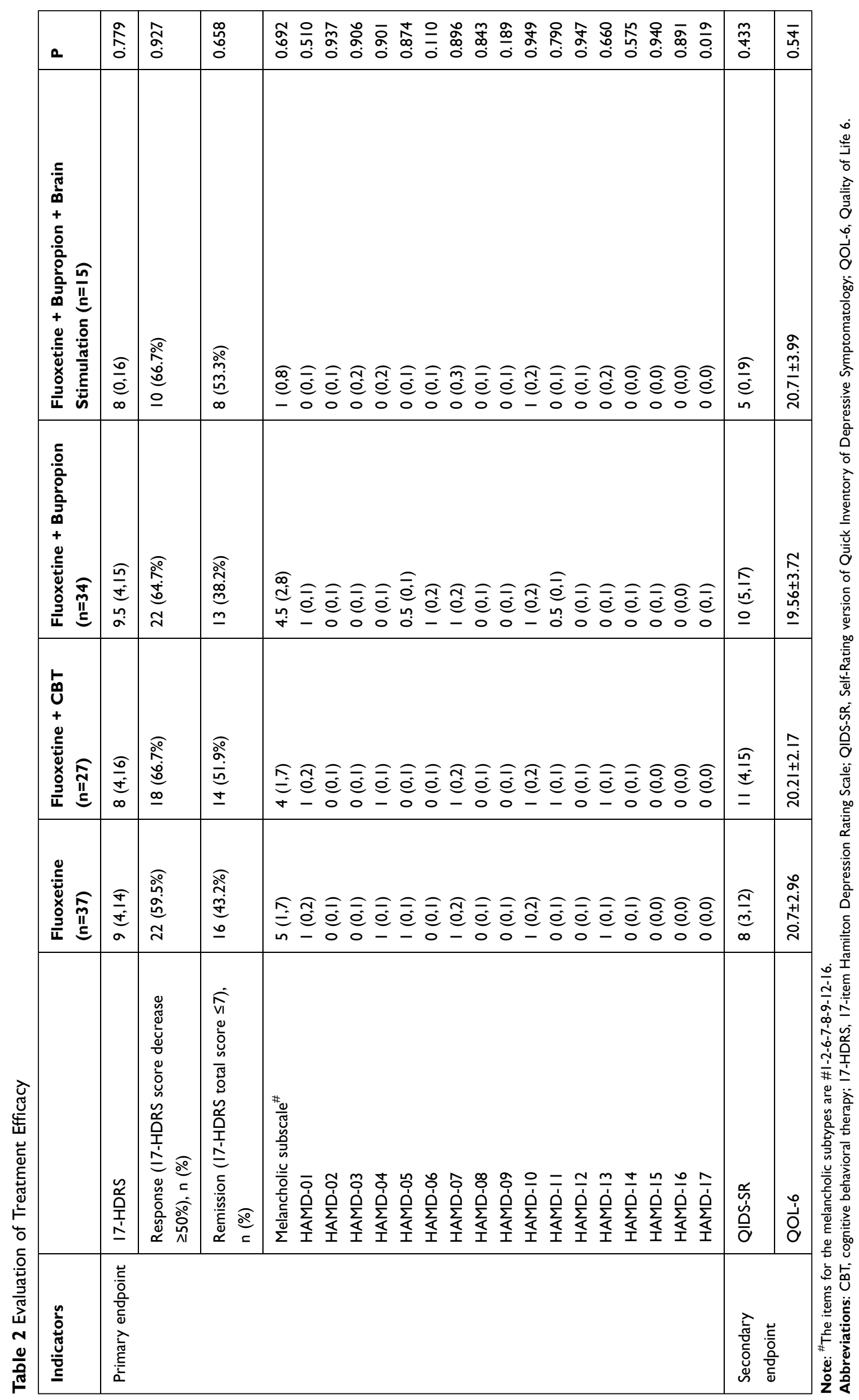



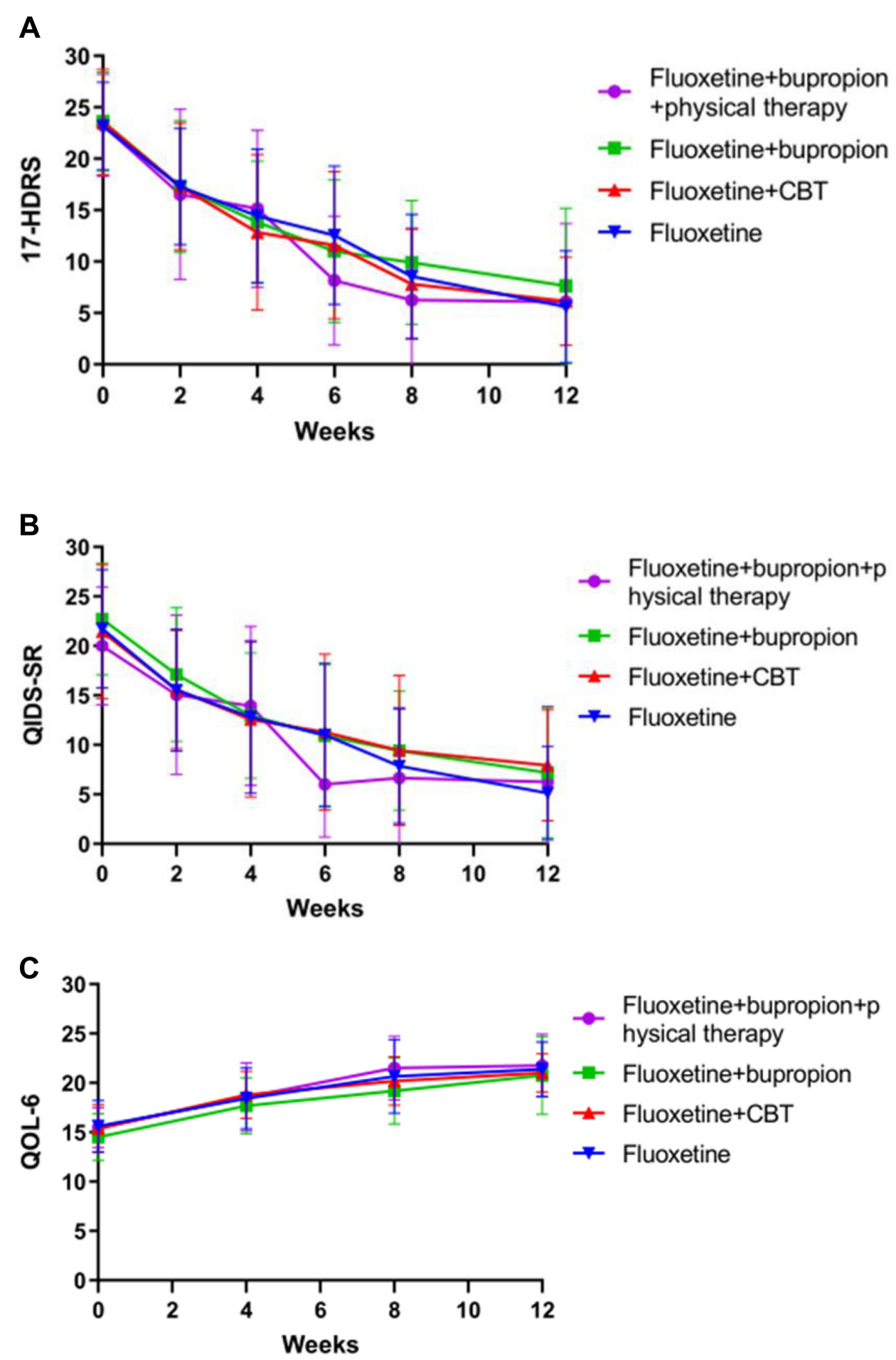

Figure I Treatment effect in each group. The treatment effect in each group was plotted using time as the $\mathrm{x}$-axis (0, 2, 4, 6, 8, and I2 weeks) and the indicators. (A) I7-item Hamilton Depression Rating Scale (I7-HDRS). (B) The self-rating version of the Quick Inventory of Depressive Symptomatology (QIDS-SR). (C) Quality of Life 6 (QOL-6).

as with cognitive impairment due to intrusive thoughts. ${ }^{26,27}$ Therefore, decreasing self-consciousness could lead to a better quality of life, but additional studies are necessary to determine whether this difference might be clinically relevant.
Fluoxetine, bupropion, MECT, and rTMS are considered safe and well-tolerated. ${ }^{3,4}$ Nevertheless, as could be expected, more AEs were reported for the fluoxetine +bupropion+physical therapies since this group was exposed to the cumulative risk of AEs from all three 
Table 3 Adverse Events in the Four Groups

\begin{tabular}{|c|c|c|c|c|c|}
\hline Indicators & $\begin{array}{l}\text { Fluoxetine } \\
(n=37)\end{array}$ & $\begin{array}{l}\text { Fluoxetine + } \\
\text { CBT }(n=27)\end{array}$ & $\begin{array}{l}\text { Fluoxetine + } \\
\text { Bupropion }(n=34)\end{array}$ & $\begin{array}{l}\text { Fluoxetine + Bupropion + } \\
\text { Brain Stimulation } \\
(n=15)\end{array}$ & $\mathbf{P}$ \\
\hline Adverse events, n (\%) & 0 & $3(11.1 \%)$ & $5(14.7 \%)$ & $2(13.3 \%)$ & 0.053 \\
\hline Gastrointestinal dysfunction & 0 & $2(7.4 \%)$ & $2(5.9 \%)$ & I (6.7\%) & 0.303 \\
\hline Abnormal liver functions & 0 & 0 & 0 & 0 & I \\
\hline Headache & 0 & 0 & $3(8.8 \%)$ & $2(13.3 \%)$ & 0.035 \\
\hline Fatigue & 0 & 0 & I (2.9\%) & 0 & 0.673 \\
\hline Allergy & 0 & 0 & 0 & 0 & I \\
\hline Tremor & 0 & 0 & I (2.9\%) & I (6.7\%) & 0.217 \\
\hline Tachycardia/ bradycardia & 0 & 0 & 0 & 0 & I \\
\hline Selfharm & 0 & I (3.7\%) & 0 & I (6.7\%) & 0.136 \\
\hline Common & 0 & 0 & 0 & 0 & l \\
\hline
\end{tabular}

Abbreviation: $\mathrm{CBT}$, cognitive behavioral therapy.

treatments. This could also explain the small number of patients who completed the treatment in this group.

This study has limitations. This was a preliminary trial with a small sample size, which was not confirmed by a power analysis. In addition, the drop-out rate was high, especially in the group receiving combined physical therapies.

In conclusion, the results suggest that fluoxetine, fluoxetine+CBT, fluoxetine+bupropion, and fluoxetine+bupropion +brain stimulation achieved similar response and remission rates in patients with melancholic depression, except that fluoxetine alone had a better effect on self-consciousness that fluoxetine+bupropion. The safety profile was manageable. Further studies should be performed to confirm those results. A non-inferiority trial might be necessary.

\section{Acknowledgments}

The authors acknowledge the help of all the study participants.

\section{Funding}

This study was funded by the National Key Research and Development Project (2016YFC1307100).

\section{Disclosure}

All authors declare that they have no conflicts of interest for this work.

\section{References}

1. Otte C, Gold SM, Penninx BW, et al. Major depressive disorder. Nat Rev Dis Primers. 2016;2:16065. doi:10.1038/nrdp.2016.65
2. Davidson JR. Major depressive disorder treatment guidelines in America and Europe. J Clin Psychiatry. 2010;71(Suppl E1):e04. doi:10.4088/JCP.9058se1c.04gry

3. American Psychiatric Association. Practice Guideline for the Treatment of Patients with Major Depressive Disorder. 3rd ed. Philadelphia: American Psychiatric Association; 2010.

4. Thase ME. The multifactorial presentation of depression in acute care. J Clin Psychiatry. 2013;74(Suppl 2):3-8. doi:10.4088/ JCP.12084su1c.01

5. Gili M, Roca M, Armengol S, Asensio D, Garcia-Campayo J, Parker G. Clinical patterns and treatment outcome in patients with melancholic, atypical and non-melancholic depressions. PLoS One. 2012;7(10):e48200. doi:10.1371/journal.pone.0048200

6. Driessen E, Hollon SD. Cognitive behavioral therapy for mood disorders: efficacy, moderators and mediators. Psychiatr Clin North Am. 2010;33(3):537-555. doi:10.1016/j.psc.2010.04.005

7. Patel K, Allen S, Haque MN, Angelescu I, Baumeister D, Tracy DK. Bupropion: a systematic review and meta-analysis of effectiveness as an antidepressant. Ther Adv Psychopharmacol. 2016;6(2):99-144. doi: $10.1177 / 2045125316629071$

8. Lisanby SH. Electroconvulsive therapy for depression. N Engl J Med. 2007;357(19):1939-1945. doi:10.1056/NEJMct075234

9. Ottosson JO, Odeberg H. Evidence-based electroconvulsive therapy. Acta Psychiatr Scand. 2012;125(3):177-184. doi:10.1111/j.16000447.2011.01812.x

10. Lefaucheur JP, Andre-Obadia N, Antal A, et al. Evidence-based guidelines on the therapeutic use of repetitive transcranial magnetic stimulation (rTMS). Clin Neurophysiol. 2014;125(11):2150-2206. doi:10.1016/j.clinph.2014.05.021

11. Bersani FS, Minichino A, Enticott PG, et al. Deep transcranial magnetic stimulation as a treatment for psychiatric disorders: a comprehensive review. Eur Psychiatry. 2013;28(1):30-39. doi:10.1016/j.eurpsy.2012.02.006

12. American Psychiatric Association. Diagnostic and Statistical Manual of Mental Disorders (DSM-5). Philadelphia: American Psychiatric Association; 2013.

13. Bobo WV, Anglero GC, Jenkins G, Hall-Flavin DK, Weinshilboum R, Biernacka JM. Validation of the 17-item Hamilton depression rating scale definition of response for adults with major depressive disorder using equipercentile linking to clinical global impression scale ratings: analysis of pharmacogenomic research network Antidepressant Medication Pharmacogenomic study (PGRN-AMPS) data. Hum Psychopharmacol. 2016;31(3):185-192. doi:10.1002/hup.2526 
14. Rush AJ, Trivedi MH, Ibrahim HM, et al. The 16-item Quick Inventory Of Depressive Symptomatology (QIDS), clinician rating (QIDS-C), and self-report (QIDS-SR): a psychometric evaluation in patients with chronic major depression. Biol Psychiatry. 2003;54 (5):573-583. doi:10.1016/s0006-3223(02)01866-8

15. Zheng YP, Zhao JP, Phillips M, et al. Validity and reliability of the Chinese hamilton depression rating scale. $\mathrm{Br} J$ Psychiatry. 1988;152:660-664. doi:10.1192/bjp.152.5.660

16. Liu J, Xiang YT, Wang G, et al. Psychometric properties of the Chinese versions of the Quick Inventory of Depressive Symptomatology - Clinician Rating (C-QIDS-C) and Self-Report (C-QIDS-SR). J Affect Disord. 2013;147(1-3):421-424. doi:10.1016/j.jad.2012.08.035

17. Valerio MP, Szmulewicz AG, Martino DJ. A quantitative review on outcome-to-antidepressants in melancholic unipolar depression. Psychiatry Res. 2018;265:100-110. doi:10.1016/j.psychres.2018.03.088

18. Joyce PR, Mulder RT, Luty SE, et al. Patterns and predictors of remission, response and recovery in major depression treated with fluoxetine or nortriptyline. Aust $N Z$ J Psychiatry. 2002;36 (3):384-391. doi:10.1046/j.1440-1614.2002.01026.x

19. Lonnqvist J, Sihvo S, Syvalahti E, Kiviruusu O. Moclobemide and fluoxetine in atypical depression: a double-blind trial. J Affect Disord. 1994;32(3):169-177. doi:10.1016/0165-0327(94)90015-9

20. Sogaard J, Lane R, Latimer P, et al. A 12-week study comparing moclobemide and sertraline in the treatment of outpatients with atypical depression. J Psychopharmacol. 1999;13(4):406-414. doi:10.1177/026988119901300412

21. Blier P, Ward HE, Tremblay P, Laberge L, Hebert C, Bergeron R. Combination of antidepressant medications from treatment initiation for major depressive disorder: a double-blind randomized study. $\mathrm{Am}$ J Psychiatry. 2010;167(3):281-288. doi:10.1176/appi.ajp.2009.09020186
22. Ionescu DF, Niciu MJ, Richards EM, Zarate CA. Pharmacologic treatment of dimensional anxious depression: a review. Prim Care Companion CNS Disord. 2014;16(3). doi:10.4088/PCC.13r01621

23. Hayes SC, Hofmann SG. The third wave of cognitive behavioral therapy and the rise of process-based care. World Psychiatry. 2017;16(3):245-246. doi:10.1002/wps.20442

24. Churchill R, Moore TH, Furukawa TA, et al. 'Third wave' cognitive and behavioural therapies versus treatment as usual for depression. Cochrane Database Syst Rev. 2013;10:CD008705. doi:10.1002/ 14651858.CD008705.pub2

25. Moret C. Combination/augmentation strategies for improving the treatment of depression. Neuropsychiatr Dis Treat. 2005;1 (4):301-309.

26. Calamari JE, Wiegartz PS, Riemann BC, et al. Obsessive-compulsive disorder subtypes: an attempted replication and extension of a symptom-based taxonomy. Behav Res Ther. 2004;42(6):647-670. doi:10.1016/S0005-7967(03)00173-6

27. Marker CD, Calamari JE, Woodard JL, Riemann BC. Cognitive self-consciousness, implicit learning and obsessive-compulsive disorder. J Anxiety Disord. 2006;20(4):389-407. doi:10.1016/j. janxdis.2005.03.003

28. Esposito CM, Buoli M. The biological face of melancholia: are there any reliable biomarkers for this depression subtype? J Affect Disord. 2020;266:802-809. doi:10.1016/j.jad.2020.02.036

29. Mutz J, Vipulananthan V, Carter B, et al. Comparative efficacy and acceptability of non-surgical brain stimulation for the acute treatment of major depressive episodes in adults: systematic review and network meta-analysis. BMJ. 2019:364. doi:10.1136/bmj.11079
Neuropsychiatric Disease and Treatment

\section{Publish your work in this journal}

Neuropsychiatric Disease and Treatment is an international, peerreviewed journal of clinical therapeutics and pharmacology focusing on concise rapid reporting of clinical or pre-clinical studies on a range of neuropsychiatric and neurological disorders. This journal is indexed on PubMed Central, the 'PsycINFO' database and CAS, and is the official journal of The International Neuropsychiatric Association (INA). The manuscript management system is completely online and includes a very quick and fair peer-review system, which is all easy to use. Visit http://www.dovepress.com/testimonials.php to read real quotes from published authors. 